\title{
Risk Factor Management in Non-Metropolitan Patients with Coronary and Peripheral Artery Disease - A Protocol of a Prospective, Multi- Center, Quality Improvement Strategy
}

\author{
Claudia Zemmrich ${ }^{\prime}$ \\ Peter Bramlage ${ }^{2}$ \\ Philipp Hillmeister ${ }^{3}$ \\ Mesud Sacirovic ${ }^{3}$ \\ Ivo Buschmann ${ }^{3}$ \\ 'Deutsche Angiologie-CRO, Berlin, \\ Germany; ${ }^{2}$ Institute for Pharmacology \\ and Preventive Medicine, Cloppenburg, \\ Germany; ${ }^{3}$ Department of Angiology, \\ Medical University Brandenburg, \\ Brandenburg, Germany
}

\begin{abstract}
Introduction: Coronary artery disease (CAD) and peripheral arterial disease (PAD) account for significant morbidity and mortality in Germany and are more prevalent in rural, nonmetropolitan areas. The goal of this study is to screen patients for their current atherosclerotic status, initiate treatment according to the latest scientific findings using a standardised multimodal approach and track their atherosclerotic status over one year.

Methods and Analysis: This manuscript describes the study protocol of a prospective, multicentre registry of 500 sequential patients with $\mathrm{CAD}$ and/or PAD in rural, non-metropolitan regions of Germany. Patients, who visit the "WalkByLab" at the Brandenburg Medical School, Brandenburg, Germany, will be assessed by using our structured, multimodal risk factor management (SMART) tool to evaluate cardiovascular morbidity data, collect information on care and deliver multimodal therapy. The study's primary objective is a cross-sectional examination of the risk profile, diagnostic and therapeutic status in this patient group. Secondary objectives include the assessment of risk factor correlations as well as changes in risk-factor profile and therapy adherence. Patients will be examined at baseline and followed up at three-monthly intervals for one year. Over this time, atherosclerotic risk factors and patient adherence to defined therapeutic strategies will be evaluated. Study completion is estimated to be December 2021. An expansion of this concept into other rural, non-metropolitan neighbouring regions is planned.

Ethics and Dissemination: This registry was assessed and approved by the ethics committee of the Brandenburg State Medical Association, Brandenburg, Germany, and conducted in accordance with the Declaration of Helsinki. The study findings will be disseminated through usual academic channels including meeting presentations and peer-reviewed publications.
\end{abstract}

Protocol Version: 1.0 .

Keywords: coronary artery disease, peripheral arterial disease, atherosclerosis, rural, risk factor, quality improvement

\section{Introduction}

\section{Background and Rationale}

Coronary artery disease (CAD) is the single most common cause of death in the developed world; accounting for approximately one in five deaths worldwide. ${ }^{1}$ In Germany, this percentage is much higher and in 2012 CAD was responsible for $43.9 \%$ of deaths in women and $36.1 \%$ deaths in men. ${ }^{2,3}$ Furthermore, it is associated with a lifetime prevalence of between 10 and $16 \%$, depending on geographical location
Correspondence: Claudia Zemmrich Deutsche Angiologie CRO (DA-CRO), Straubinger Strasse 20, Berlin, 12683, Germany

Tel +49 I78 7I52405

Email claudia.zemmrich@da-cro.de 
within the country, and accounts for the largest single portion of treatment costs. ${ }^{3}$ Due to the high morbidity, mortality and socioeconomic importance of $\mathrm{CAD}$, the timely diagnosis and initiation of cost-effective treatment is essential. ${ }^{1}$

Peripheral artery disease (PAD) denotes vascular diseases caused by atherosclerosis of the abdominal aorta, iliac and lower-extremity arteries leading to stenosis or occlusion. ${ }^{4}$ Patients with PAD can experience a wide range of medical issues including claudication, ischaemic rest pain, ischaemic ulcerations, repeated hospitalisations, revascularisations and limb loss. This, in return, reduces the patient's quality of life (QoL) and can lead to depression. ${ }^{4}$ The prevalence of PAD is increasing worldwide and is strongly age-related. Overall, an estimated $20 \%$ of the German population above 70 years of age are affected with this condition. ${ }^{5}$ Guidelines for the treatment and management of PAD are now in place in Germany. ${ }^{5}$

The co-existence of both CAD and PAD in patients was first discovered in $1960 .^{6}$ The prevalence of CAD in patients with PAD varies widely in published reports, partially due to the definition CAD ( $\geq 50 \%$ or $\geq 70 \%$ diameter stenosis), the method of diagnosis and the location of the PAD. ${ }^{7}$ However, more than half of patients suffering from PAD that are approximately 80 years of age with, will also have concomitant $\mathrm{CAD}{ }^{8,9}$ Understanding the relationship between CAD and PAD is essential as CADrelated complications are the leading cause of morbidity and mortality in patients undergoing surgery for PAD. ${ }^{6}$

While epidemiology data provide an overview of the incidence and prevalence of CAD and PAD across Germany as a whole, various studies have shown that there is significant disparity in the rates of both CAD and PAD within the country. ${ }^{3,10-14}$ A key question is why does a developed country have such a variation in CAD and PAD rates? It is speculated that these sparsely populated, rural, non-metropolitan regions (eg, Brandenburg, Mecklenburg-West Pomerania, Saxony and Saxony-Anhalt) have poor accessibility to medical expertise and patients would have to undergo long journeys to attend specialist medical care centres for treatment. This is compounded by ineffective communication between the large number of specialists that patients may visit and the lack of standardised treatment approaches for the different manifestations of both CAD and PAD.

To address these differences between German states, there is an urgent need for lasting prevention strategies that address societal, behavioural and clinical aspects of these diseases. Therefore, data on the risk factor profile of patients with both CAD and PAD are needed to develop treatment strategies for these patients and to provide adequate secondary intervention.

\section{Ethics and Dissemination}

This registry was assessed and approved by the ethics committee of the Brandenburg State Medical Association, Brandenburg, Germany and conducted in accordance with the Declaration of Helsinki. The study findings will be disseminated through usual academic channels including meeting presentations and peerreviewed publications.

\section{Aims and Objectives}

For this prospective analysis, we developed a structured, multimodal risk factor management (SMART) tool for patients with known or newly diagnosed CAD or PAD to evaluate cardiovascular morbidity data and to collect care information in rural, non-metropolitan regions of Germany.

The aims of this study are to: 1) Screen patients for their current atherosclerotic status; 2) Initiate therapy according to the latest scientific findings using a standardised multimodal approach and 3) Evaluate the effectiveness of therapy over a one-year timeframe.

It is hoped that the SMART tool will provide a standardised approach to the management and treatment of patients with $\mathrm{CAD}$ and/or PAD in rural, non-metropolitan areas of Germany and promote communication between medical specialists treating this patient group.

\section{Methods and Analysis}

\section{Study Setting}

This study is based on a prospective, multicentre registry of patients with $\mathrm{CAD}$ and/or PAD who visit the "WalkByLab" at the Brandenburg Medical School, Brandenburg, Germany. The "WalkByLab" is a laboratory providing comprehensive cardiovascular diagnostics for patients with suspected cardiovascular diseases. Diagnostic tools include echocardiography, duplex ultrasound of all vascular beds, treadmill testing, ECG, laboratory examination including a wide range of investigational parameters, flow mediated dilatation, ankle-brachial-index $(\mathrm{ABI})$, oxygen-to-see $8 \quad(\mathrm{O} 2 \mathrm{C})$ and quality-of-lifequestionnaires.

Patients, athletes and other interested parties reporting any physical symptoms associated with either walking or 
running, can enter the "WalkByLab" and will pass through the diagnostic program at baseline and after 1 year of follow-up. An expansion of this concept into other rural, non-metropolitan neighbouring regions is planned in the long-term.

\section{Patient Eligibility Criteria}

Patients attending the "WalkByLab" will be recruited in a sequential manner with the following inclusion criteria: Age of at least 18 years, with a proven history of CAD or PAD defined as $\geq 50 \%$ coronary (angiographic) or peripheral arterial (aortal, infra-aortal or carotid) stenosis (angiographic or duplex ultrasound) or any anamnestic PCI or cardiovascular surgery or ankle brachial index $(\mathrm{ABI})<0.9$ with claudication-related walking distance restriction categorized by the Rutherford Classification. Patients will be excluded from the study if they have a life-expectancy of less than one year, do not provide or are unable to provide consent for the study or are unable to follow medical instructions regarding therapeutic indications. Written informed consent must be provided prior to enrolment.

\section{Objectives}

The primary objective of this study is a cross-sectional examination of the risk profile, diagnostic and therapeutic status in consecutive, unselected patients with CAD and/or PAD in rural, non-metropolitan regions of Germany.

Study secondary objectives include the change in riskfactor profile one-year after implementation of the SMART program; therapy adherence to standardised multimodal risk factor management over one-year (acceptable $\geq 70 \%$, poor $<70 \%$ ); correlation of risk factors and therapy adherence with cardiovascular events; and differences between patient cohorts "CAD" and "PAD" regarding sociodemographic and medical parameters, as well as therapeutic strategies.

\section{Data Collection}

\section{Patient Characteristics and Medical History}

At baseline, demographic information (date of documentation, sex and year of birth) will be collected. A history of vascular and other diseases/risk factors, medications (dosage, dose interval) and weekly sports activity will be captured as well as current New York Heart Association (NYHA) grade, cardiac arrhythmia, presence of current/ past malignancies, European Society of Cardiology (ESC) score, CHADSVASC score, HASBLED score, health-related Quality-of-Life (QoL) using the Short
Form 36 (SF-36) and Pain Disability Index (PDI) will be recorded.

\section{Laboratory Analyses}

Blood will be collected for laboratory analysis (full blood count, total cholesterol, high-density lipoprotein [HDL]cholesterol, low-density lipoprotein [LDL]-cholesterol, triglycerides, lipoprotein A [lp(a)], blood glucose, HbA1c, $\mathrm{N}$-terminal pro brain natriuretic protein (nt-pro-BNT), high-sensitivity troponin $\mathrm{T}$ [HsTnT], estimated glomerular filtration rate [eGFR] and growth differentiation factor 15 [GDF-15]).

\section{Diagnostic Examinations}

Patients will undergo a physical examination (height, weight, blood pressure and heart rate).

Diagnostic examinations include echocardiography for determination of left ventricular ejection fraction (LVEF) with the SIMPSON method, duplex ultrasound of extracranial carotid arteries for intima-media-thickness, plaque burden and abdominal aorta for aneurysm and plaque detection, ECG, measurement of flow mediated dilatation (AngioDefender, EveristHealth, USA), "oxygen-to-see" (O2C)(LEA Medical technique, Germany), ankle-brachial index (ABI), pulse wave index (PWI), pain-free and absolute walking distance.

\section{Follow-Up Visits}

At months three, six and nine ( \pm one month) additional patient data will be captured either in a face-to-face (F2F) meeting or over the telephone. Due to the COVID-19 pandemic, the majority of Month 3, 6, 9 follow-up visits will be performed by telephone calls to avoid direct patient contact. Any medical events that have been occurred since the baseline visit will be recorded (including but not limited to arterial hypertension, dyslipidemia, diabetes mellitus, myocardial infarction, coronary angioplasty/stent implantation, coronary bypass surgery, lower limb amputation, aortic aneurysm, clinically relevant bleeding and non-cardiovascular medical events), current NYHA grade, changes in medication since baseline (and reason for this change) and intervention by the family doctor to stabilise blood pressure, cholesterol, HbAlc and/or blood glucose.

\section{Month Final Visit}

At month 12 ( \pm one month or as soon later as the COVID-19 pandemic allowed to investigate elective 
patients), the final patient visit including a blood sample will be collected for laboratory analysis to measure LDL-cholesterol, HbA1c, nt-pro-BNP and eGFR. A medical history of events since the previous visit will capture information on the presence of new comorbidities, current NYHA grade, health-related QoL (using SF-36 and PDI), measurement of ABI, PWI, FMD, O2C and pain-free and absolute walking distance, echocardiography for LVEF, duplex ultrasound of carotid arteries and aorta for IMD and presence of plaques and changes in medication (including reason for change). See Figure 1 for the study procedure flowchart.

Data collection will be monitored by a subcontracted clinical research organisation, the German Angiological Clinical Research organization (DA-CRO). Monitoring visits will cover approximately $10 \%$ of the study patients. If necessary, these visits will be supplemented by telephone or written communication.

\section{Statistics}

The study aims at enrolling 500 patients. The number of participants is based on the intention to record changes in treatment after one-year of follow-up for patients with varying risk factors and assumes a patient dropout rate of $20-30 \%$. To keep the drop-out rate as low as possible we recall patients up to three times in case of a missed visit and call their family doctors to investigate if potential outcome events occurred. All patients agree at study entry to forward their family doctors' contact data.

The primary biometric evaluation of all data collected is descriptive. The results will be presented in tabular and graphical form based on the predefined evaluation plan. The number of patients, mean value plus standard deviation (SD) for normally distributed continuous variables, median plus range will be determined for non-normally distributed continuous variables, and frequencies and percentage frequencies will be determined for categorical variables.
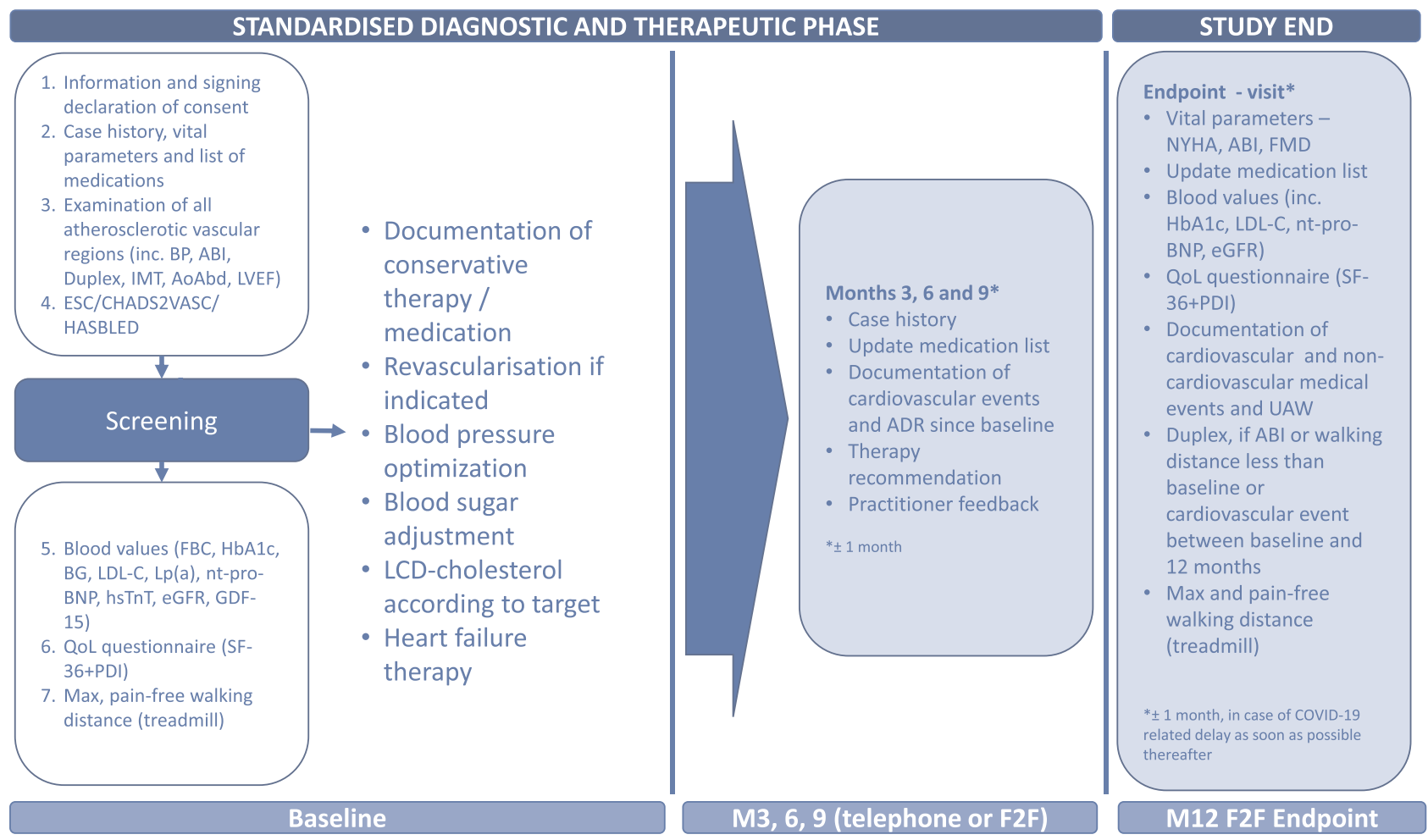

Figure I Study overview.

Abbreviations: $A B I$, ankle brachial index; AoAbd, aorta abdominalis; FBC, full blood count; BG, blood glucose; CHADS2VASC, calculator for atrial fibrillation; eGFR, estimated glomerular filtration rate; ESC, European Society of Cardiology; F2F, face-to-face; FMD, flow mediated dilation; GDF-I5, growth differentiation factor I5; HASBLED, calculator for bleeding risk; HsTnT, high-sensitivity troponin T; IMT, intima media thickness; LDL-C, low-density lipoprotein cholesterol; Lp(a), lipoprotein (a); LVEF, left ventricular ejection fraction; nt-pro-BNP, N-terminal pro brain natriuretic protein; NYHA, New York Heart Association; PDI, Pain Disability Index; BP, blood pressure; SF-36, Short Form (36) Health Survey; ADR, adverse drug reactions. 
Mean, Median and proportions will be reported with $95 \%$ confidence intervals. Prevalence rate ratios with 95\% confidence intervals ( $95 \%$ CIs) will be calculated using logbinomial models (SPSS Statistics software version 24.0 (Armonk, NY. IBM Corporation), log-link function and binomial distribution function). Kaplan-Meier analyses will be used for survival rates and freedom from major cardiovascular complications (eg, stroke, embolism, bleeding, hospitalization for vascular intervention or amputation) using Log rank tests. Logistic regression analyses will be performed to evaluate outcome predictors of mortality and cardiovascular events. Hazard ratios will be calculated by Cox-regression and adjusted for major baseline variables. Comparisons between groups will be carried out using the Student's $t$-test with Levine's homogeneity of variance or the MannWhitney $U$-test for continuous variables, as appropriate, and a Fishers' exact or Chi-square test for categorical variables. We plan to calculate all regression models with adjustment for groups of coronary or peripheral artery disease only and combined and for LDL-cholesterol target and lipoprotein (a) level groups. A two-Tailed $P$-value of $<0.05$ will be considered statistically significant.

\section{Discussion}

The prevalence of CAD in Germany, and the disparities between the regions, have been well documented. ${ }^{3}$ Interestingly, however, PAD is often under-recognised and, therefore, under-treated; ${ }^{15}$ often leading to longer-term complications and a lower QoL for patients. ${ }^{16}$ It is known that prevalence of PAD increases with age and, in Germany as a whole, an estimated 20 and $17 \%$ of men and women over the age of 65 years have PAD, and the incidence is higher still in patients with diabetes. ${ }^{17,18}$ There is limited, if any, information on the prevalence of PAD in rural areas of Germany and the results of our study will fill this void.

Inequalities in publicly provided services, such as healthcare facilities, have been widely documented between rural and urban areas both worldwide and in Germany. ${ }^{19}$ These differences can have substantial socioeconomic impacts. In Germany, various strategies are currently under investigation to improve healthcare provision in rural areas, such as the introduction of trained medical assistants and telemedicine (telephone-based medical provision). ${ }^{20}$ Studies have also confirmed that mobility and accessibility of services is strongly linked to wellbeing in rural areas, thus supporting the need for good, localised provision of healthcare. ${ }^{21}$ The introduction and effectiveness of SMART, which will be established in rural areas, might help to redress the current urban/rural balance for patients with CAD and/or PAD and could, in the longer term, be rolled out across Germany to promote the vascular wellbeing of patients in all states.

In Germany, patient documentation is separated according to inpatient and outpatient care and a common electronic health record is not yet in place. Furthermore, data from trials, such as this one, are rarely interfaced with patient records. $^{22}$ The Medical Informatics Initiative, established in November 2015, is actively trying to promote the sharing of clinical research and patient information. It is hoped that the findings of our study will promote better communication and patient-information sharing between healthcare specialists and support existing strategies for data-sharing.

Overall, we hope that this study will have a positive impact on the awareness of CAD and PAD co-appearance and improve risk factor management in a rural, nonmetropolitan area of Germany.

\section{Data Sharing Statement}

There are no data in this work.

\section{Ethics Approval}

This research involves human subjects and has received approval from the ethics committee of the Brandenburg State Medical Association.

\section{Acknowledgments}

The authors thank Andrea Bilitza and Jeanett Förster for their logistic contribution to establish and run the "WalkByLab" including outstanding efforts to maintain patients' compliance throughout the Follow-Up visits.

\section{Author Contributions}

All authors made a significant contribution to the work reported, whether that is in the conception, study design, execution, acquisition of data, analysis and interpretation, or in all these areas; took part in drafting, revising or critically reviewing the article; gave final approval of the version to be published; have agreed on the journal to which the article has been submitted; and agreed to be accountable for all aspects of the work.

\section{Funding}

This work was supported with an unrestricted grant from Bayer Vital GmbH.

\section{Disclosure}

The authors report no conflicts of interest in this work. 


\section{References}

1. Cassar A, Holmes DR, Rihal CS, et al. Chronic coronary artery disease: diagnosis and management. Mayo Clin Proc. 2009;84 (12):1130-1146. doi:10.4065/mcp.2009.0391

2. Plass D, Vos T, Hornberg $\mathrm{C}$, et al. Trends in disease burden in Germany: results, implications and limitations of the Global Burden of Disease study. Dtsch Arztebl Int. 2014;111(38):629-638. doi:10.3238/arztebl.2014.0629

3. Dornquast C, Kroll LE, Neuhauser HK, et al. Regional differences in the prevalence of cardiovascular disease. Dtsch Arztebl Int. 2016;113 (42):704-711. doi:10.3238/arztebl.2016.704

4. Olin JW, Sealove BA. Peripheral artery disease: current insight into the disease and its diagnosis and management. Mayo Clin Proc. 2010;85(7):678-692. doi:10.4065/mcp.2010.0133

5. Lawall H, Huppert P, Espinola-Klein C, et al. German guideline on the diagnosis and treatment of peripheral artery disease a comprehensive update 2016. Vasa. 2017;46(2):79-86. doi:10.1024/0301-1526/a000603

6. Juergens JL, Barker NW, Hines EA. Arteriosclerosis obliterans: review of 520 cases with special reference to pathogenic and prognostic factors. Circulation. 1960;21:188-195. doi:10.1161/01. cir.21.2.188

7. Hur DJ, Kizilgul M, Aung WW, et al. Frequency of coronary artery disease in patients undergoing peripheral artery disease surgery. Am J Cardiol. 2012;110(5):736-740. doi:10.1016/j.amjcard.2012.04.059

8. Aronow WS, Ahn C. Prevalence of coexistence of coronary artery disease, peripheral arterial disease, and atherothrombotic brain infarction in men and women $>$ or $=62$ years of age. Am $J$ Cardiol. 1994;74(1):64-65. doi:10.1016/0002-9149(94)90493-6

9. Ness J, Aronow WS. Prevalence of coexistence of coronary artery disease, ischemic stroke, and peripheral arterial disease in older persons, mean age 80 years, in an academic hospital-based geriatrics practice. J Am Geriatr Soc. 1999;47(10):1255-1256. doi:10.1111/ j.1532-5415.1999.tb05208.x

10. Stang A, Stang M. An inter-state comparison of cardiovascular risk factors in Germany: towards an explanation of high ischemic heart disease mortality in Saxony-Anhalt. Dtsch Arztebl Int. 2014;111(31-32):530-536. doi:10.3238/arztebl.2014.0530

11. Muller-Nordhorn J, Rossnagel K, Mey W, et al. Regional variation and time trends in mortality from ischaemic heart disease: east and West Germany 10 years after reunification. J Epidemiol Community Health. 2004;58(6):481-485. doi:10.1136/jech.2003.013367
12. Dornquast C, Willich SN, Reinhold T. Prevalence, mortality, and indicators of health care supply-association analysis of cardiovascular diseases in Germany. Front Cardiovasc Med. 2018;5:158. doi: $10.3389 /$ fcvm.2018.00158

13. Lampert T, Müters S, Kuntz B, et al. 30 years after the fall of the Berlin Wall: regional health differences in Germany. $J$ Health Monit. 2019.

14. Bhatt DL, Steg PG, Ohman EM, et al. International prevalence, recognition, and treatment of cardiovascular risk factors in outpatients with atherothrombosis. JAMA. 2006;295(2):180-189. doi:10.1001/jama.295.2.180

15. Hirsch AT, Criqui MH, Treat-Jacobson D, et al. Peripheral arterial disease detection, awareness, and treatment in primary care. JAMA. 2001;286(11):1317-1324. doi:10.1001/jama.286.11.1317

16. Regensteiner JG, Hiatt WR, Coll JR, et al. The impact of peripheral arterial disease on health-related quality of life in the peripheral arterial disease awareness, risk, and treatment: new resources for survival (PARTNERS) program. Vasc Med. 2008;13(1):15-24. doi: $10.1177 / 1358863 \times 07084911$

17. Diehm C, Schuster A, Allenberg JR, et al. High prevalence of peripheral arterial disease and co-morbidity in 6880 primary care patients: cross-sectional study. Atherosclerosis. 2004;172(1):95-105. doi:10.1016/s0021-9150(03)00204-1

18. Lange S, Diehm C, Darius H, et al. High prevalence of peripheral arterial disease and low treatment rates in elderly primary care patients with diabetes. Exp Clin Endocrinol Diabetes. 2004;112 (10):566-573. doi:10.1055/s-2004-830408

19. Potrafke N, Roesel F. The urban-rural gap in healthcare infrastructure: does government ideology matter? Reg Stud. 2019;1-12. doi:10.1080/00343404.2019.1623390

20. Kuhn B, Kleij KS, Liersch S, et al. Which strategies might improve local primary healthcare in Germany? An explorative study from a local government point of view. BMC Fam Pract. 2017;18 (1):105. doi:10.1186/s12875-017-0696-Z

21. Camarero L, Oliva J. Thinking in rural gap: mobility and social inequalities. Palgrave Commun. 2019;5(1). doi:10.1057/s41599019-0306-x

22. Gehring S, Eulenfeld R. German medical informatics initiative: unlocking data for research and health care. Methods Inf Med. 2018;57(S 01):e46-e49. doi:10.3414/ME18-13-0001
Vascular Health and Risk Management

\section{Publish your work in this journal}

Vascular Health and Risk Management is an international, peerreviewed journal of therapeutics and risk management, focusing on concise rapid reporting of clinical studies on the processes involved in the maintenance of vascular health; the monitoring, prevention and treatment of vascular disease and its sequelae; and the involvement of metabolic disorders, particularly diabetes. This journal is indexed on PubMed Central and MedLine. The manuscript management system is completely online and includes a very quick and fair peerreview system, which is all easy to use. Visit http://www.dovepress. com/testimonials.php to read real quotes from published authors. 